\title{
ON THE DUAL OF AN EXPONENTIAL SOLVABLE LIE GROUP
}

\author{
BRADLEY N. CURREY
}

\begin{abstract}
Let $G$ be a connected, simply connected exponential solvable Lie group with Lie algebra $\mathfrak{g}$. The Kirillov mapping $\eta: \mathfrak{g}^{*} / \operatorname{Ad}^{*}(G) \rightarrow \hat{G}$ gives a natural parametrization of $\hat{G}$ by co-adjoint orbits and is known to be continuous. In this paper a finite partition of $\mathfrak{g}^{*} / \operatorname{Ad}^{*}(G)$ is defined by means of an explicit construction which gives the partition a natural total ordering, such that the minimal element is open and dense. Given $\pi \in \hat{G}$, elements in the enveloping algebra of $g_{c}$ are constructed whose images under $\pi$ are scalar and give crucial information about the associated orbit. This information is then used to show that the restriction of $\eta$ to each element of the above-mentioned partition is a homeomorphism.
\end{abstract}

1. Introduction. Let $G$ be a real, connected, simply connected exponential solvable Lie group with Lie algebra $\mathfrak{g}$. By a representation of $G$ we shall mean a strongly continuous, unitary representation of $G$ in some Hilbert space, and we denote the dual of $G$ by $\hat{G}$, that is, the set of unitary equivalence classes of topologically irreducible representation of $G$. Denote by $\eta$ the natural mapping of the set $\mathfrak{g}^{*} / \operatorname{Ad}^{*}(G)$ of co-adjoint orbits in the dual $\mathfrak{g}^{*}$ of $\mathfrak{g}$ onto $\hat{G}$. When $\mathfrak{g}^{*} / \operatorname{Ad}^{*}(G)$ is given the quotient topology and $\hat{G}$ the hull kernel topology, $\eta$ is continuous. It was first conjectured by A. A. Kirillov in [8] and proved by I. Brown in [3] that if $G$ is nilpotent, $\eta$ is a homeomorphism. K. Joy in a later paper [7] gives a much shorter proof of Brown's Theorem using results of J. M. G. Fell pertaining to the space $S(G)$ of subgroup representation pairs $(\pi, H)$, where $H$ is a closed connected subgroup of $G$ and $\pi$ is an unitary equivalence class of representations of $H$. Two results on the bicontinuity of $\eta$ when $G$ is exponential are due to J. Boidol [2] and H. Fujiwara [6]. Boidol shows that $\eta^{-1}$ is continuous provided that $G$ is $*$-regular; *-regularity is seen to fail however even for a completely solvable group of dimension four. On the other hand, Fijuwara proves the existence of a dense open subset $U$ of $\hat{G}$ such that $V=\eta^{-1}(U)$ is dense and such that the restriction of $\eta$ to $V$ is a homeomorphism. However, Fujiwara's result provides no explicit characterization of $U$. Finally, it is known that $\eta$ is a homeomorphism for all $G$ of dimension less than six. Those cases which are not $*$-regular are handled by constructing elements $W$ in the center of the enveloping algebra $U\left(\mathfrak{g}_{c}\right)$, and using the fact that the mapping $\phi_{W}$ on $\hat{G}$ given by $\rho(W)=\phi_{W}(\rho) I$ is continuous. $\phi_{W} \circ \eta$ can be regarded as an $\operatorname{Ad}^{*}(G)$-invariant polynomial function of $\mathfrak{g}^{*}$, and as such provides enough information to conclude convergence of the corresponding orbits. In the general case the center of $U\left(\mathfrak{g}_{c}\right)$ is not large enough to yield sufficient information about $\eta^{-1}$.

Now let $\mathfrak{n}$ be the nilradical of $\mathfrak{g}$, and let $\rho \in \hat{G}$ such that $\rho$ is extended from $N=\exp (\mathfrak{n})$. A generalization of the construction mentioned above is given whereby

Received by the editors October 30, 1986 and, in revised form, June 19, 1987.

1980 Mathematics Subject Classification (1985 Revision). Primary 22E27, Secondary 17B30. 
elements $w_{i} \in U\left(\mathfrak{g}_{c}\right)$ are constructed such that $\left\{\rho\left(W_{i}\right)\right\}$ are scalar operators whose values allow one to systematically obtain $\eta^{-1}(\rho)$ from the orbit of $\left.\rho\right|_{N}$. The Kirillov mapping has a natural generalization in the context of the space of subgroup representation pairs $(\rho, H)$ such that $H \supset N$ and $\rho \in \hat{H}$, and a theorem regarding this mapping is proved which has as a corollary the following. There is a finite partition $\left\{U_{\alpha}\right\}$ of $\mathfrak{g}^{*} / \operatorname{Ad}^{*}(G)$-obtained by an explicit construction depending only on a choice of Jordan-Hölder sequence for $\eta$ - on each element of which $\eta$ is open.

2. Preliminaries. Let $\mathfrak{g}$ be a real, solvable Lie algebra of exponential type. For any subspace $\mathfrak{h}$ of $\mathfrak{g}$, let $\mathfrak{h}^{*}$ denote the dual space of $\mathfrak{h}$, and if $\mathfrak{j}$ is a subspace of $\mathfrak{g}$ such that $[\mathfrak{h}, \mathfrak{h}] \subset \mathfrak{j}$ and $\lambda \in \mathfrak{j}^{*}$, denoted by $B_{\lambda}$ the bilinear form defined on $\mathfrak{h}$ by $B_{\lambda}(X, Y)=\lambda([X, Y]), X, Y \in \mathfrak{h}$. For any subset $\mathfrak{s}$ of $\mathfrak{h}$, denote by $\mathfrak{s}^{\lambda, \mathfrak{h}}$ the orthogonal complement of $\mathfrak{s}$ in $h$ with respect to $B_{\lambda}$. The radical $\mathfrak{h}^{\lambda, \mathfrak{h}}$ of $B_{\lambda}$ will also be denoted by $R(\lambda, \mathfrak{h})$.

Let $\left\{\mathfrak{h}_{n}\right\}_{n=1}^{\infty}$ be a sequence of subspaces of $\mathfrak{g}$. We shall say that $\mathfrak{h}_{n}$ converges to a subspace $\mathfrak{h}$ (or write $\mathfrak{h}_{n} \rightarrow \mathfrak{h}$ ) if there are positive integers $K$ and $d$ such that for each $n>K$, there is a basis $X_{1}^{(n)}, X_{2}^{(n)}, \ldots, X_{d}^{(n)}$ of $\mathfrak{h}_{n}$ and a basis $X_{1}, X_{2}, \ldots, X_{d}$ of $\mathfrak{h}$ with $X_{j}=\lim _{n} X_{j}^{(n)}, 1 \leq j \leq d$. Suppose that $\mathfrak{h}_{n} \rightarrow \mathfrak{h}$, and let $W_{n} \in \mathfrak{h}_{n}$, $n \geq 1$, such that for some $W \in \mathfrak{g}, W=\lim _{n} W_{n}$. Then $W \in h$, and it follows that if for some $\mathfrak{h}^{\prime}, \mathfrak{h}_{n} \rightarrow \mathfrak{h}^{\prime}$, then $\mathfrak{h}^{\prime}=\mathfrak{h}$, and if $\mathfrak{h}_{n}$ is a subalgebra (ideal) for infinitely many $n$, then $\mathfrak{h}$ is a subalgebra (ideal). Clearly every sequence $\left\{\mathfrak{h}_{n}\right\}$ of nontrivial subspaces of $\mathfrak{g}$ has a subsequence which converges, and it is easily seen that $\mathfrak{h}_{n} \rightarrow \mathfrak{h}$ if and only if every convergent subsequence of $\left\{\mathfrak{h}_{n}\right\}$ converges to $\mathfrak{h}$.

LEMMA 2.1. Let $\left\{\mathfrak{j}_{n}\right\}_{n=1}^{\infty}$ be a sequence of subspaces of $\mathfrak{g}$ such that for each $n$, $\mathfrak{j}_{n} \subset \mathfrak{h}_{n}$, and suppose that $\mathfrak{j}_{n} \rightarrow \mathfrak{j}$ and $\mathfrak{h}_{n} \rightarrow \mathfrak{h}$. Let $\left\{\lambda_{n}\right\}_{n=1}^{\infty}$ be a sequence in $\mathfrak{g}^{*}$ such that for some $\lambda \in \mathfrak{g}^{*},\left.\lambda\right|_{[\mathfrak{g}, \mathfrak{g}]}=\left.\lim _{n} \lambda_{n}\right|_{[\mathfrak{g}, \mathfrak{g}]}$, and $\operatorname{dim}_{\mathbf{R}}\left(\mathfrak{j}^{\lambda, \mathfrak{h}}\right)=\liminf _{n} \operatorname{dim}_{\mathbf{R}}\left(\mathfrak{j}_{n}^{\lambda_{n}, \mathfrak{h}_{n}}\right)$. Then $\mathrm{j}_{n}^{\lambda_{n}, \mathfrak{h}_{n}} \rightarrow \mathfrak{j}^{\lambda, \mathfrak{h}}$.

ProOF. Let $K$ and $d$ be positive integers such that for each $n>K$, there is a basis $Y_{1}^{(n)}, Y_{2}^{(n)}, \ldots, Y_{d}^{(n)}$ of $\mathrm{j}_{n}$ with $\lim _{n} Y_{j}^{(n)}=Y_{j}, 1 \leq j \leq d$, and $\left\{Y_{j}\right\}$ a basis of j. Let $\left\{\mathfrak{j}_{k}^{\lambda_{k}, h_{k}}\right\}$ be any convergent subsequence, $\mathfrak{j}_{k}^{\lambda_{k}, h_{k}} \rightarrow \mathfrak{j}_{0}$, and let $W \in \mathfrak{j}_{0}$. Then for each $k$, there is $W^{(k)} \in \mathfrak{j}_{k}^{\lambda_{k}, h_{k}}$ such that $W=\lim _{k} W^{(k)}$, and we have

$$
\lambda\left(\left[W, Y_{j}\right]\right)=\lim _{k} \lambda_{k}\left(\left[W^{(k)}, Y_{j}^{(k)}\right]\right)=0,
$$

$1 \leq j \leq d$. Thus $\mathfrak{j}_{0} \subset \mathfrak{j}_{\lambda, \mathfrak{h}}$. On the other hand,

$$
\operatorname{dim}_{\mathbf{R}}\left(\mathfrak{j}_{0}\right) \geq \lim _{n} \inf _{\operatorname{dim}_{\mathbf{R}}}\left(\mathfrak{j}_{n}^{\lambda_{n}, \mathfrak{h}_{n}}\right)
$$

so that $\mathfrak{j}_{0}=\mathfrak{j}^{\lambda, \mathfrak{h}}$, and hence $\mathfrak{j}_{n}^{\lambda_{n}, \mathfrak{h}_{n}} \rightarrow \mathfrak{j}^{\lambda, \mathfrak{h}}$.

Let $\mathfrak{h}$ be a subalgebra of $\mathfrak{g}$. We denote by $U(\mathfrak{h})$ the enveloping algebra of $\mathfrak{h}$ and regard $U(\mathfrak{h})$ as a subalgebra of $U(\mathfrak{g})$. We denote the complexification $\mathfrak{h} \otimes_{\mathbf{R}} \mathbf{C}$ by $\mathfrak{h}_{c}$ and regard $U\left(\mathfrak{h}_{c}\right)$ as a subalgebra of $U\left(\mathfrak{g}_{c}\right)$.

Let $G$ be a connected, simply connected Lie group with Lie algebra $\mathfrak{g}$, and let $H$ be the closed, connected subgroup of $G$ with Lie algebra $\mathfrak{h}$. Denote by $\eta_{H}$ Kirillov mapping $\mathfrak{h}^{*} / \operatorname{Ad}^{*}(H) \rightarrow \hat{H}$, let $\pi$ be a representation of $H$, and let $\lambda \in \mathfrak{h}^{*}$. We shall say that $\pi$ corresponds to $\lambda$ if $\pi \in \eta_{H}\left(\operatorname{Ad}^{*}(H) \lambda\right)$. If $\mathfrak{p} \in \mathfrak{h}^{*}$ is a polarization at $\lambda$, we occasionally use the notation $\operatorname{ind}(\lambda, \mathfrak{p})$ for the irreducible representation 
$\operatorname{ind}\left(\chi_{\lambda}, P, H\right)$ of $H$ induced by the character $\chi_{\lambda}$ of $P=\exp (\mathfrak{p})$ with differential $i\left(\left.\lambda\right|_{\mathfrak{p}}\right)$.

Now let $\lambda \in \mathfrak{g}^{*}$, and let $\mathfrak{m}$ be a nilpotent subalgebra of $\mathfrak{g}$.

DEFINITION 2.2. A pair $\left(\mathfrak{m}_{1}, \mathfrak{m}_{0}\right)$ of $\mathfrak{m}$-ideals such that $\mathfrak{m}_{0} \subset \mathfrak{m}_{1}, \operatorname{dim}_{\mathbf{R}}\left(\mathfrak{m}_{1} / \mathfrak{m}_{0}\right)$ $=1, \mathfrak{m}_{0} \subset R(\lambda, \mathfrak{m})$ and $\mathfrak{m}_{1} \not \subset R(\lambda, \mathfrak{m})$ will be called a Kirillov pair in $\mathfrak{m}$ at. $\lambda$.

Let $\left(\mathfrak{m}_{1}, \mathfrak{m}_{0}\right)$ be a Kirillov pair in $\mathfrak{m}$ at $\lambda$, and let $\mathfrak{l}=\mathfrak{m}_{1}^{\lambda, \mathfrak{m}}$. Then $\mathfrak{l}$ is a codimension 1 subalgebra of $\mathfrak{m}$. Let $\pi_{1}$ be an irreducible representation of $L=\exp (\mathfrak{l})$ corresponding to $\left.\lambda\right|_{\mathfrak{l}}$, and let $X \in \mathfrak{m} \sim \mathfrak{l}$. Then the representation $\pi=\pi\left(\pi_{1}, X\right)$ defined in $L^{2}\left(\mathbf{R}, H\left(\pi_{1}\right)\right)$ by the formula

$$
(\pi(y \exp s X) f)(t)=\pi_{1}(\exp t X y \exp -t X) f(t+s) \quad(y \in L, s, t \in \mathbf{R})
$$

corresponds to $\left.\lambda\right|_{\mathfrak{m}}$. The primary representation $\tilde{\pi}_{1}$ defined in $L^{2}\left(\mathbf{R}, H\left(\pi_{1}\right)\right)$ by $\left(\tilde{\pi}_{1}(y) f\right)(t)=\pi_{1}(y) f(t), y \in L$, can be differentiated in the space $C^{\infty}(\pi)$ of smooth vectors for $\pi$, that is, $C^{\infty}\left(\tilde{\pi}_{1}\right) \supset C^{\infty}(\pi)$. The following lemma is more or less well known, but crucial in this paper.

LEMMA 2.3. There is an explicit construction by which, given any $W \in U\left(\mathfrak{l}_{c}\right)$, one obtains $\tilde{W} \in U\left(\mathfrak{l}_{c}\right)$ such that $\pi(\tilde{W})=\tilde{\pi}_{1}(W)$.

PROOF. Let $m$ be a positive integer such that ad $X^{m+1} \equiv 0$, and let $W \in U\left(\mathfrak{l}_{c}\right)$. We construct an element $W_{m} \in U\left(\mathfrak{l}_{c}\right)$ as follows. Let (by abuse of notation) $t$ denote the operator on $C^{\infty}(\pi)$ defined by $\phi(t) \rightarrow t \phi(t)$. We have $\pi(W)=$ $\sum_{j=0}^{m}\left(t^{j} / j !\right) \tilde{\pi}_{1}\left(\operatorname{ad} X^{j} W\right)$ so that $\pi\left(\operatorname{ad} X^{m} W\right)=\tilde{x}_{1}\left(\operatorname{ad} X^{m} W\right)$. Let $Y$ be the element in $\mathfrak{m}_{1} \sim \mathfrak{m}_{0}$ such that $\lambda(Y)=0$, and $B_{\lambda}(X, Y)=1$, so that $\pi(Y)=i t$. Define $W_{1} \in U\left(\mathfrak{l}_{c}\right)$ by

$$
W_{1}=W-\frac{\operatorname{ad} X^{m} W \cdot(-i Y)^{m}}{m !} .
$$

Then $\pi\left(W_{1}\right)=\sum_{j=0}^{m-1}\left(t^{j} / j !\right) \pi_{1}\left(\operatorname{ad} X^{j} W\right)$ and $\pi\left(\operatorname{ad} X^{m-1} W_{1}\right)=\tilde{\pi}_{1}\left(\operatorname{ad} X^{m-1} W\right)$. If $m>1$, set

$$
W_{2}=W_{1}-\frac{\operatorname{ad} X^{m-1} W_{1} \cdot(-i Y)^{m-1}}{(m-1) !}
$$

and we find that $\pi\left(\operatorname{ad} X^{m-2} W_{2}\right)=\tilde{\pi}_{1}\left(\operatorname{ad} X^{m-2} W\right)$. Continue in this way until $W_{m}=\tilde{W}$ is obtained. Q.E.D.

3. A partition of the dual of a nilpotent Lie group. Now let us assume that $\mathfrak{g}$ is nilpotent; fix $\lambda \in \mathfrak{g}^{*}$. By induction on the dimension of $\mathfrak{g}$ it is easily seen that there is a sequence of subalgebras $\mathfrak{g}=\mathfrak{m}_{0} \supset \mathfrak{m}_{1} \supset \cdots \supset \mathfrak{m}_{d}$ satisfying the conditions

(i) $\mathfrak{m}_{d}$ is a polarization at $\lambda$.

(ii) If $R(\lambda, \mathfrak{g}) \neq \mathfrak{g}$, then $d>0$ and for each $k=1,2, \ldots, d-1$, there is a Kirillov pair $\left(\mathfrak{m}_{k 1}, \mathfrak{m}_{k 0}\right)$ in $\mathfrak{m}_{k}$ at $\lambda$ such that $\mathfrak{m}_{k+1}=\mathfrak{m}_{k 1}^{\lambda, \mathfrak{m}_{k}}$. Thus if $R(\lambda, \mathfrak{g}) \neq \mathfrak{g}$, then $\left.d=\frac{1}{2} \operatorname{dim}\left(\operatorname{Ad}^{*}(G)\right) \lambda\right)$.

DEFINITION 3.1. A sequence of subalgebras satisfying conditions (i) and (ii) above will be called a Kirillov sequence for $\lambda$ in $\mathfrak{g}$.

Let $d$ be a nonnegative integer. Let us say that an operator $D$ on $C^{\infty}\left(\mathbf{R}^{d}\right)$ $\left(C^{\infty}\left(\mathbf{R}^{0}\right) \equiv \mathbf{C}\right)$ is a polynomial differential operator if there is a polynomial $P$ in $2 d$ indeterminants with complex coefficients such that

$$
D=P\left(t_{1}, \ldots, t_{d}, \partial / \partial t_{1}, \ldots, \partial / \partial t_{d}\right) .
$$


A theorem of Kirillov (cf. [8, Theorem 7.1]) states that $\eta\left(\operatorname{Ad}^{*}(G) \lambda\right)$ has a realization $\pi$ in a space of functions on $\mathbf{R}^{d}$ such that the image of $U\left(\mathfrak{g}_{c}\right)$ under $\pi$ is the set of polynomial differential operators. In this section we shall determine when it is possible, given a sequence $\left\{\lambda_{n}\right\}_{n=1}^{\infty}$ in $\mathfrak{g}^{*}$ such that $\lambda_{n} \rightarrow \lambda=\lambda_{0}$, to obtain a corresponding sequence $\left\{\pi_{n}\right\}_{n=0}^{\infty}$ of irreducible representations such that given any $D$ as above, there is a sequence $\left\{W_{n}\right\}_{n=0}^{\infty}$ in $U\left(\mathfrak{g}_{c}\right)^{(m)}$ for some $m$, with $W_{n} \rightarrow W_{0}$ and $\pi_{n}\left(W_{n}\right)=D$ for each $n$.

Let $\mathfrak{g}=\mathfrak{g}_{p} \supset \mathfrak{g}_{p-1} \supset \cdots \supset \mathfrak{g}_{0}=(0)$ be a Jordan-Hölder sequence for $\mathfrak{g}$. Define subsets $e(\lambda), j(\lambda)$ and $i(\lambda)$ of $\{1,2, \ldots, p\}$ as follows. Set

$$
e(\lambda)=\left\{t \mid \mathfrak{g}_{t}+R(\lambda, \mathfrak{g}) \supsetneqq \mathfrak{g}_{t-1}+R(\lambda, \mathfrak{g})\right\}
$$

and let $\mathfrak{p}(\lambda)=\sum_{t} R\left(\lambda, \mathfrak{g}_{t}\right)$. Define $j(\lambda) \subset e(\lambda)$ by

$$
j(\lambda)=\left\{t \mid \mathfrak{g}_{t}+\mathfrak{p}(\lambda) \supsetneqq \mathfrak{g}_{t-1}+\mathfrak{p}(\lambda)\right\}
$$

and let $i(\lambda)=e(\lambda) \sim j(\lambda)$. Then $\operatorname{card}(e(\lambda))=\operatorname{dim}\left(\operatorname{Ad}^{*}(G) \lambda\right)$ and it is shown in [1] that $\mathfrak{p}(\lambda)$ is a polarization at $\lambda$, hence $\operatorname{card}(j(\lambda))=\frac{1}{2} \operatorname{card}(e(\lambda))$. If $e(\lambda) \neq\{\phi\}$, we shall write $e(\lambda)=\left\{e_{1}<e_{2}<\cdots<e_{2 d}\right\}$. We define a sequence of subalgebras $\mathfrak{g}=\mathfrak{g}^{0}(\lambda) \supset \mathfrak{g}^{1}(\lambda) \supset \cdots \supset \mathfrak{g}^{d}(\lambda)$ as follows. Setting $\mathfrak{g}^{0}(\lambda)=\mathfrak{g}$, assume that for some $k \geq 0, \mathfrak{g}^{k}(\lambda)$ is defined and $\mathfrak{g}^{k}(\lambda) \neq R\left(\lambda, \mathfrak{g}^{k}(\lambda)\right)$. Let $i_{k+1}$ be the smallest index such that $\mathfrak{g}_{i_{k+1}} \cap \mathfrak{g}^{k}(\lambda) \not \subset R\left(\lambda, \mathfrak{g}^{k}(\lambda)\right)$ and set

$$
\mathfrak{g}^{k+1}(\lambda)=\left(\mathfrak{g}_{i_{k+1}} \cap \mathfrak{g}^{k}(\lambda)\right)^{\lambda, \mathfrak{g}^{k}(\lambda)} .
$$

Note that $\mathfrak{g}^{k+1}(\lambda)$ is codimension 1 in $\mathfrak{g}^{k}(\lambda)$. If $\mathfrak{g}^{k}(\lambda)=R\left(\lambda, \mathfrak{g}^{k}(\lambda)\right)$, then let the sequence terminate at $\mathfrak{g}^{k}(\lambda)$, and set $k=d$. Thus $\mathfrak{g}^{d}(\lambda)$ is isotropic with respect to $B_{\lambda}$. If $e(\lambda) \neq\{\phi\}$, then in this way we obtain a sequence of indices $i_{1}, i_{2}, \ldots, i_{d}$. Note that if $R(\lambda, \mathfrak{g}) \subset \mathfrak{g}^{k}(\lambda)$, then $R(\lambda, \mathfrak{g}) \subset \mathfrak{g}^{k+1}(\lambda), 0 \leq k \leq d$; thus we have

$$
R(\lambda, \mathfrak{g}) \subset R\left(\lambda, \mathfrak{g}^{k}(\lambda)\right) \subset \mathfrak{g}^{k}(\lambda), \quad 0 \leq k \leq d .
$$

Now for each $k=1,2, \ldots, d$, let $j_{k}$ be the smallest index such that $\mathfrak{g}_{j_{k}} \cap \mathfrak{g}^{k-1}(\lambda) \not \subset$ $\mathfrak{g}^{k}(\lambda)$.

LEMMA 3.2. For each $k=1,2,3, \ldots, d, i_{k} \in e(\lambda)$ and $j_{k} \in e(\lambda)$. If $k<d$, $i_{k}<i_{k+1}$, and for $k \leq d, i_{k}<j_{k}$. Moreover $\mathfrak{g}^{d}(\lambda)=\mathfrak{p}(\lambda), i(\lambda)=\left\{i_{k}\right\}_{k=1}^{d}$, and $j(\lambda)=\left\{j_{k}\right\}_{k=1}^{d}$.

PROOF. If $i_{k} \notin e(\lambda)$, there is $Y \in R(\lambda, \mathfrak{g})$ such that $\mathfrak{g}_{i_{k}}=\mathbf{R} Y+\mathfrak{g}_{i_{k}-1}$. But since $R(\lambda, \mathfrak{g}) \subset R\left(\lambda, \mathfrak{g}^{k-1}(\lambda)\right)$,

$$
\mathfrak{g}_{i_{k}} \cap \mathfrak{g}^{k-1}(\lambda)=\mathbf{R} Y+\left(\mathfrak{g}_{i_{k}-1} \cap \mathfrak{g}^{k-1}(\lambda)\right) \subset R\left(\lambda, \mathfrak{g}^{k-1}(\lambda)\right)
$$

a contradiction. If $j_{k} \notin e(\lambda)$, let $X \in R(\lambda, \mathfrak{g})$ such that $\mathfrak{g}_{j_{k}}=\mathbf{R} X+\mathfrak{g}_{j_{k}-1}$. Since $R(\lambda, \mathfrak{g}) \subset \mathfrak{g}^{k}(\lambda)$,

$$
\mathfrak{g}_{j_{k}} \cap \mathfrak{g}^{k-1}(\lambda)=\mathbf{R} X+\left(\mathfrak{g}_{j_{k}-1} \cap \mathfrak{g}^{k-1}(\lambda)\right) \subset \mathfrak{g}^{k}(\lambda)
$$

a contradiction. This proves the first statement of the lemma.

Now by definition of $i_{k}$,

$$
\mathfrak{g}_{i_{k}} \cap \mathfrak{g}^{k-1}(\lambda)=\mathbf{R} Y+\mathfrak{g}_{i_{k}-1} \cap \mathfrak{g}^{k-1}(\lambda) \subset \mathbf{R} Y+R\left(\lambda, \mathfrak{g}^{k-1}(\lambda)\right) .
$$


Thus

$$
\begin{gathered}
{\left[\mathfrak{g}_{i_{k}} \cap \mathfrak{g}^{k-1}(\lambda), \mathfrak{g}_{i_{k}} \cap \mathfrak{g}^{k-1}(\lambda)\right] \subset\left[Y, R\left(\lambda, \mathfrak{g}^{k-1}(\lambda)\right)\right]} \\
+\left[R\left(\lambda, \mathfrak{g}^{k-1}(\lambda)\right), R\left(\lambda, \mathfrak{g}^{k-1}(\lambda)\right)\right] \subset \operatorname{Ker}(\lambda) .
\end{gathered}
$$

By definition of $\mathfrak{g}^{k}(\lambda)$ and $j_{k}$, it follows that $\mathfrak{g}_{i_{k}} \cap \mathfrak{g}^{k-1}(\lambda) \subset \mathfrak{g}^{k}(\lambda)$, and hence that $i_{k}<j_{k}$, and that

$$
\mathfrak{g}_{i_{k}} \cap \mathfrak{g}^{k}(\lambda)=\mathfrak{g}_{i_{k}} \cap \mathfrak{g}^{k-1}(\lambda) \subset R\left(\lambda, \mathfrak{g}^{k}(\lambda)\right)
$$

therefore $i_{k}<i_{k+1}$.

Next we show that $\mathfrak{g}^{d}(\lambda)=\mathfrak{p}(\lambda)$. For this, let $X \in R\left(\lambda, \mathfrak{g}_{t}\right)$, and suppose that $X \in \mathfrak{g}^{k}(\lambda)$ for some $k<d$. We show that $X \in \mathfrak{g}^{k+1}(\lambda)$. Suppose $t<i_{k+1}$. By choice of $i_{k+1}, X \in \mathfrak{g}_{t} \cap \mathfrak{g}^{k}(\lambda) \subset R\left(\lambda, \mathfrak{g}^{k}(\lambda)\right) \subset \mathfrak{g}^{k+1}(\lambda)$. Suppose $t \geq i_{k+1}$; then $X \in R\left(\lambda, \mathfrak{g}_{t}\right) \cap \mathfrak{g}^{k}(\lambda) \subset\left(\mathfrak{g}_{t} \cap \mathfrak{g}^{k}(\lambda)\right)^{\lambda, \mathfrak{g}^{k}(\lambda)} \subset \mathfrak{g}^{k+1}(\lambda)$. Since $X \in \mathfrak{g}^{0}(\lambda)=\mathfrak{g}$, it follows that $X \in \mathfrak{g}^{d}(\lambda)$.

Let us show now that $i(\lambda)=\left\{i_{k}\right\}_{k=1}^{d}$. Note that for each $k, R\left(\lambda, \mathfrak{g}^{k}(\lambda)\right) \subset$ $\mathfrak{g}^{k+1}(\lambda)$ so that $R\left(\lambda, \mathfrak{g}^{k}(\lambda)\right) \subset R\left(\lambda, \mathfrak{g}^{k+1}(\lambda)\right)$ and hence $R\left(\lambda, \mathfrak{g}^{k}(\lambda)\right) \subset \mathfrak{p}(\lambda)$. Thus $\mathfrak{g}_{i_{k}} \subset \mathfrak{g}_{i_{k}-1}+\mathfrak{p}(\lambda)$ and $i_{k} \notin j(\lambda)$. It follows that $i(\lambda)=\left\{i_{k}\right\}_{k=1}^{d}$.

Finally, to see that $\left\{j_{k}\right\}_{k=1}^{d}=j(\lambda)$, note that $j_{k} \in j(\lambda)$, since if not, then $\mathfrak{g}_{j_{k}} \subset \mathfrak{g}_{j_{k}-1}+\mathfrak{g}^{k}(\lambda)$, hence $\mathfrak{g}_{j_{k}} \cap \mathfrak{g}^{k-1}(\lambda) \subset\left(\mathfrak{g}_{j_{k}-1}+\mathfrak{g}^{k}(\lambda)\right) \cap \mathfrak{g}^{k-1}(\lambda)=\mathfrak{g}_{j_{k}-1} \cap$ $\mathfrak{g}^{k-1}(\lambda)+\mathfrak{g}^{k}(\lambda)=\mathfrak{g}^{k}(\lambda)$ a contradiction.

Let $j \in j(\lambda)$, and let $k_{0}$ be the smallest $k, 1 \leq k \leq d$, such that $\mathfrak{g}_{j} \subset \mathfrak{g}_{j-1}+\mathfrak{g}^{k}(\lambda)$. We claim that $j=j_{k_{0}}$. Now $\mathfrak{g}_{j} \subset \mathfrak{g}_{j-1}+\mathfrak{g}^{k_{0}-1}(\lambda)$, so we may write $\mathfrak{g}_{j}=\mathbf{R} X+\mathfrak{g}_{j-1}$ where $X \in \mathfrak{g}^{k_{0}-1}(\lambda)$, and by choice of $k_{0}, X \in \mathfrak{g}^{k_{0}}(\lambda)$. Hence $\mathfrak{g}_{j} \cap \mathfrak{g}^{k_{0}-1}(\lambda) \subset \mathfrak{g}^{k_{0}}(\lambda)$ and $j \geq j_{j_{k_{0}}}$, by choice of $j \geq j_{j_{k_{0}}}$. If $j>j_{k_{0}}$, then choose $\tilde{X} \in \mathfrak{g}_{j_{k_{0}}}$ such that $\mathfrak{g}^{k_{0}-1}(\lambda)=\mathbf{R} \tilde{X}+\mathfrak{g}^{k_{0}}(\lambda)$. Since $\operatorname{dim}\left(\mathfrak{g}^{k_{0}-1}(\lambda) / \mathfrak{g}^{k_{0}}(\lambda)\right)=1$, there are elements $a \neq 0$, $b \neq 0$ in such that $W=a \tilde{X}+b X \in \mathfrak{g}^{k_{0}}(\lambda)$. But $\mathfrak{g}_{j}=\mathbf{R} W+\mathfrak{g}_{j-1} \subset \mathfrak{g}_{j-1}+\mathfrak{g}^{k_{0}}(\lambda)$, contradicting our choice of $k_{0}$; therefore $j=j_{k_{0}}$, and the proof of the lemma is finished.

Now let $E$ denote the set of pairs

$$
E=\left\{(e(\lambda), j(\lambda)) \mid \lambda \in \mathfrak{g}^{*}\right\}
$$

and for $d$ a positive integer, let

$$
E_{d}=\{(e, j) \in E \mid \operatorname{card}(j)=d\} .
$$

Let us regard elements of $E_{d}$ as ordered $3 d$-tuples of integers

$$
(e, j)=\left(e_{1}, e_{2}, \ldots, e_{2 d}, j_{1}, j_{2}, \ldots, j_{d}\right)
$$

where $e_{1}<e_{2}<\cdots$, and $\left\{j_{1}, \ldots, j_{d}\right\}=j$ is indexed by the inductive process above. We define a total order on $E$ in the following way. Let $(\phi, \phi)$ be the maximal element of $E$, and regarding $E_{d}$ as above, let $E_{d}$ have the natural lexographic ordering. If $d>d^{\prime}$, let us say that for any $\alpha \in E_{d}, \alpha^{\prime} \in E_{d^{\prime}}, \alpha<\alpha^{\prime}$.

Now for each $\alpha \in E$, set $\Omega_{\alpha}=\left\{\lambda \in \mathfrak{g}^{*} \mid(e(\lambda), j(\lambda))=\alpha\right\}$ and for each $e_{0}=e\left(\lambda_{0}\right)$, $\Omega_{e_{0}}=\left\{\lambda \in \mathfrak{g}^{*} \mid e(\lambda)=e_{0}\right\}$ so that $\Omega_{e_{0}}=\bigcup\left\{\Omega_{(e, j)} \mid e=e_{0}\right\}$. If $s \in G$, and $\lambda \in \mathfrak{g}^{*}$, then $\mathfrak{g}^{k}\left(\operatorname{Ad}^{*}(s) \lambda\right)=\operatorname{Ad}(s)\left(\mathfrak{g}^{k}(\lambda)\right)$ and it follows that each $\alpha \in E, \Omega_{\alpha}$ is $G$-invariant. The sets $\Omega_{e_{0}}$ were first considered by Pukanszky in [10], and the sets $\Omega_{\alpha}$ are considered by N. V. Pedersen in a paper to appear. 
Let $\left\{Z_{1}, Z_{2}, \ldots, Z_{p}\right\}$ be a basis compatible with the Jordan-Hölder sequence chosen at the beginning of this section. Let $e=e\left(\lambda_{0}\right)$ for some $\lambda_{0}$, let $P_{e}^{i j}(\lambda)=$ $\lambda\left(\left[Z_{e_{i}}, Z_{e_{j}}\right]\right), e_{i}, e_{j} \in e$, and set $P_{e}(\lambda)=\operatorname{det}\left(\left(P_{e}^{i j}(\lambda)\right)\right)$. Letting the set $\left\{e(\lambda) \mid \lambda \in \mathfrak{g}^{*}\right\}$ have the total ordering inherited from $E$, it is shown in [9] that

$$
\Omega_{e}=\left\{\lambda \in \mathfrak{g}^{*} \mid P_{e^{\prime}}(\lambda)=0, e^{\prime}<e \text { and } P_{e}(\lambda) \neq 0\right\} \text {. }
$$

Now for each $e=e(\lambda)$, let $J_{e}=\{j \mid(e, j) \in E\}$, and let $J_{e}$ have the total ordering inherited from $E$.

PROPOSITION 3.3. There are polynomials $P_{(e, j)}, j \in J_{e}$, such that for each $j \in J_{e}$,

$$
\Omega_{(e, j)}=\left\{\lambda \in \Omega_{e} \mid P_{\left(e, j^{\prime}\right)}(\lambda)=0, j^{\prime}<j \text { and } P_{\left(e, j^{\prime}\right)}(\lambda) \neq 0\right\} .
$$

ProOF. Let $j \in J_{e}$, and write $j=\left\{j_{1}, \ldots, j_{d}\right\}$ and $i=e-j=\left\{i_{1}, \ldots, i_{d}\right\}$ as in the inductive process above. Let $\lambda \in \Omega_{e}$ and for each $k=1,2, \ldots, d$ set $e^{(k)}=e-\left\{i_{1}, \ldots, i_{k}, j_{1}, \ldots, j_{k}\right\}$ and define elements $Z_{t}^{k}(\lambda) \in g, t \in e^{(k)}$ as follows. Note that $\left\{Z_{t}\right\}_{t \in e}$ is a basis for $\mathfrak{g}$ modulo $R(\lambda, \mathfrak{g})$. Let $Z_{t}^{1}(\lambda)=Z_{t}$ if $t \in e^{(1)}, t<j_{1}$ and for $t>j_{1}$, set

$$
Z_{t}^{1}(\lambda)=B_{\lambda}\left(Z_{j_{1}}, Z_{i_{1}}\right) Z_{t}-B_{\lambda}\left(Z_{t}, Z_{i_{1}}\right) Z_{j_{1}} .
$$

Suppose that $\lambda \in \Omega_{\left(e, j^{\prime}\right)}$ with $j^{\prime} \geq j$ and write $j^{\prime}=\left\{j_{1}^{\prime}, \ldots, j_{d}^{\prime}\right\}, i=e-j^{\prime}=$ $\left\{i_{1}^{\prime}, \ldots, i_{d}^{\prime}\right\}$. Since $e_{1}=i_{1}=i_{1}^{\prime}$, clearly $j_{1}^{\prime}=j_{1}$ if and only if $B_{\lambda}\left(Z_{j_{1}}, Z_{i_{1}}\right) \neq 0$, and in this case, $\left\{Z_{t}^{1}(\lambda)\right\}_{t \in e^{(1)}}$ is a basis of $\mathfrak{g}^{1}(\lambda)$ modulo $R\left(\lambda, \mathfrak{g}^{1}(\lambda)\right)$. Therefore, by definition of $j_{1}, j_{2}$, we have $j_{1}^{\prime}=j_{1}$ and $j_{2}^{\prime}=j_{2}$ if and only if, $B_{\lambda}\left(Z_{j_{1}}, Z_{i_{1}}\right) \neq$ 0 and $B_{\lambda}\left(Z_{j_{2}}^{1}(\lambda), Z_{i_{2}}^{1}(\lambda)\right) \neq 0$. Now define $Z_{t}^{2}(\lambda), t \in e^{(2)}$ in the same way as $\left\{Z_{t}^{1}(\lambda)\right\}_{t \in e^{(1)}}$, so that if $j_{1}^{\prime}=j_{1}, j_{2}^{\prime}=j_{2}$, then $\left\{Z_{t}^{2}(\lambda)\right\}_{t \in e^{(2)}}$ is a basis of $\mathfrak{g}^{2}(\lambda)$ modulo $R\left(\lambda, \mathfrak{g}^{2}(\lambda)\right)$. Continuing in this way, set

$$
P_{e, j}(\lambda)=B_{\lambda}\left(Z_{j_{1}}, Z_{i_{1}}\right) B_{\lambda}\left(Z_{j_{2}}^{1}(\lambda), Z_{i_{2}}^{1}(\lambda)\right) \cdots B_{\lambda}\left(Z_{j_{d}}^{d-1}(\lambda), Z_{i_{d}}^{d-1}(\lambda)\right)
$$

and the proposition follows. Q.E.D.

COROllary 3.4. Let $\alpha_{0}$ be the minimal element of $E$. Then $\Omega_{\alpha_{0}}$ is Zariski open in $\mathfrak{g}^{*}$. Moreover, for each $\alpha \in E, \Omega_{\alpha}$ is open in $\bigcup_{\beta \geq \alpha} \Omega_{\beta}$.

PROOF. That $\Omega_{\alpha_{0}}$ is Zariski open is clear. As for the second statement, let $\alpha \in E, \alpha=(e, j)$. $\Omega_{\alpha}$ is Zariski open in $\bigcup_{j^{\prime} \geq j} \Omega_{\left(e, j^{\prime}\right)}$ by Proposition 3.3. But $\Omega_{e}$ is open in $\bigcup_{e^{\prime} \geq e} \Omega_{e^{\prime}}$, and hence $\bigcup_{j^{\prime} \geq j} \Omega_{\left(e, j^{\prime}\right)}=\Omega_{e} \cap \bigcup_{\beta \geq \alpha} \Omega_{\beta}$ is open in $\bigcup_{\beta \geq \alpha} \Omega_{\beta}$, and it follows that $\Omega_{\alpha}$ is open in $\bigcup_{\beta \geq \alpha} \Omega_{\beta}$. Q.E.D.

Now for each $\lambda \in \mathfrak{g}^{*}$ such that $\{\phi\} \neq e(\lambda)=i(\lambda) \cup j(\lambda), i(\lambda)=\left\{i_{1}, i_{2}, \ldots, i_{d}\right\}$, $j(\lambda)=\left\{j_{1}, j_{2}, \ldots, j_{d}\right\}$, define for each $0 \leq k<d, \mathfrak{m}_{k 1}(\lambda)=\mathfrak{g}^{k}(\lambda) \cap \mathfrak{g}_{i_{k+1}}$ and $\mathfrak{m}_{k 0}(\lambda)=\mathfrak{g}^{k}(\lambda) \cap \mathfrak{g}_{i_{k+1}-1}$. Then $\left(\mathfrak{m}_{k 1}(\lambda), \mathfrak{m}_{k 0}(\lambda)\right)$ is a Kirillov pair in $\mathfrak{g}^{k}(\lambda)$ at $\lambda$, and $\mathfrak{m}_{k 1}^{\lambda} \mathfrak{g}^{k(\lambda)}=\mathfrak{g}^{k+1}(\lambda), 0 \leq k<d$. Thus the sequence $\mathfrak{g}^{0}(\lambda) \supset \mathfrak{g}^{1}(\lambda) \supset \cdots \supset \mathfrak{g}^{d}(\lambda)$ is a Kirillov sequence for $\lambda$ in $\mathfrak{g}$.

THEOREM 3.5. Let $d$ be a positive integer and let $\alpha \in E_{d}$. Let $\left\{\lambda_{n}\right\}_{n=0}^{\infty}$ be a sequence in $\Omega_{\alpha}$ which converges to $\lambda_{0}$. Then for each $n \geq 0$, there is an irreducible representation $\pi_{n}$ corresponding to $\lambda_{n}$ such that if

$$
D=P\left(t_{1}, t_{2}, \ldots, t_{d}, \partial / \partial t_{1}, \partial / \partial t_{2}, \ldots, \partial / \partial t_{d}\right)
$$


is any polynomial differential operator, then there is an integer $m>0$ and a sequence $\left\{W_{n}\right\}_{n=0}^{\infty}$ in $U\left(\mathfrak{g}_{c}\right)^{(m)}$ which converges to $W_{0}$ and such that $\pi_{n}\left(W_{n}\right)=D$, $n=0,1,2, \ldots$.

Proof. Clearly we may assume that for some $1 \leq k \leq d$, either $D=t_{k}$ or $D=$ $\partial / \partial t_{k}$. For each $n \geq 0$, we have the data $\left\{\mathfrak{g}^{k}\left(\lambda_{n}\right)\right\}_{k=0}^{d},\left\{\left(\mathfrak{m}_{k 1}\left(\lambda_{n}\right), \mathfrak{m}_{k 0}\left(\lambda_{n}\right)\right)\right\}_{k=0}^{d-1}$ as in the remarks preceding the theorem. Note that for each $n \geq 0,1 \leq t \leq p$, and $1 \leq k \leq d, \operatorname{dim}\left(\mathfrak{g}_{t} \cap \mathfrak{g}^{k}\left(\lambda_{n}\right)\right)=\operatorname{card}\left(\left\{j_{s} \mid s \leq k, j_{s}<t\right\}\right)$. Now by Lemma 1.1, we have that $\mathfrak{g}^{1}\left(\lambda_{n}\right) \rightarrow \mathfrak{g}^{1}\left(\lambda_{0}\right)$. Since $\operatorname{dim}_{\mathbf{R}}\left(\mathfrak{g}_{t} \cap \mathfrak{g}^{1}\left(\lambda_{n}\right)\right)=\operatorname{dim}_{\mathbf{R}}\left(\mathfrak{g}_{t} \cap \mathfrak{g}^{1}\left(\lambda_{0}\right)\right), n=1,2,3, \ldots$, it follows that $\mathfrak{g}_{t} \cap \mathfrak{g}^{1}\left(\lambda_{n}\right) \rightarrow \mathfrak{g}_{t} \cap \mathfrak{g}^{1}\left(\lambda_{0}\right)$, and in particular, $\mathfrak{m}_{11}\left(\lambda_{n}\right) \rightarrow \mathfrak{m}_{11}\left(\lambda_{0}\right)$ and $\mathfrak{m}_{10}\left(\lambda_{n}\right) \rightarrow \mathfrak{m}_{10}\left(\lambda_{0}\right)$. But then Lemma 1.1 implies that $\mathfrak{g}^{2}\left(\lambda_{n}\right) \rightarrow \mathfrak{g}^{2}\left(\lambda_{0}\right)$. Continuing in this way, we obtain for each $k=0,1, \ldots, d-1, \mathfrak{g}^{k+1}\left(\lambda_{n}\right) \rightarrow \mathfrak{g}^{k+1}\left(\lambda_{0}\right)$, $\mathfrak{m}_{k 1}\left(\lambda_{n}\right) \rightarrow \mathfrak{m}_{k 1}\left(\lambda_{0}\right)$ and $\mathfrak{m}_{k 0}\left(\lambda_{n}\right) \rightarrow \mathfrak{m}_{k 0}\left(\lambda_{0}\right)$. Now, for each $0 \leq k \leq d, n \geq 0$, we shall define an irreducible representation $\pi_{k}^{(n)}$ of $G^{k}\left(\lambda_{n}\right)=\exp _{G}\left(\mathfrak{g}^{k}\left(\lambda_{n}\right)\right)$. Let $\pi_{d}^{(n)}$ be the character of $G^{d}\left(\lambda_{n}\right)$ with differential $i\left(\lambda_{n} \mid \mathfrak{g}_{d}\left(\lambda_{n}\right)\right)$. Choose $X_{d}^{(0)} \in$ $\mathfrak{g}^{d-1}\left(\lambda_{0}\right) \sim \mathfrak{g}^{d}\left(\lambda_{0}\right)$, and since $\mathfrak{g}^{d-1}\left(\lambda_{n}\right) \rightarrow \mathfrak{g}^{d-1}\left(\lambda_{0}\right)$ and $\mathfrak{g}^{d}\left(\lambda_{n}\right) \rightarrow \mathfrak{g}^{d}(\lambda)$, we can choose $X_{d}^{(n)} \in \mathfrak{g}^{d-1}\left(\lambda_{n}\right) \sim \mathfrak{g}^{d}\left(\lambda_{n}\right), n=1,2,3, \ldots$, such that $X_{d}^{(n)} \rightarrow X_{d}^{(0)}$. Now for each $n$, define $\pi_{d-1}^{(n)}=\pi\left(\pi_{d}^{(n)}, X_{d}^{(n)}\right)$ as in formula (1) above, that is, for each $f \in L^{2}\left(\mathbf{R}, H\left(\pi_{d}^{(n)}\right)\right)=L^{2}(\mathbf{R}), y \in G^{d}\left(\lambda_{n}\right)$, and $s, t \in \mathbf{R}$,

$$
\left(\pi_{d-1}^{(n)}\left(y \cdot \exp _{G}\left(s X_{d}^{(n)}\right)\right) f\right)(t)=\pi_{d}^{(n)}\left(\exp _{G}\left(t X_{d}^{(n)}\right) \cdot y \cdot \exp \left(-t X_{d}^{(n)}\right)\right) f(t+s) .
$$

We continue in this way, choosing $X_{k}^{(0)} \in \mathfrak{g}^{k-1}\left(\lambda_{0}\right) \sim \mathfrak{g}^{k}\left(\lambda_{0}\right)$ and $X_{k}^{(n)} \in \mathfrak{g}^{k-1}\left(\lambda_{n}\right)$ $\sim \mathfrak{g}^{k}\left(\lambda_{n}\right), n=1,2,3, \ldots$, such that $X_{k}^{n} \rightarrow X_{k}^{(0)}$, for each $k$, so that

$$
H\left(\pi_{k}^{(n)}\right)=L^{2}\left(\mathbf{R}, H\left(\pi_{k+1}^{(n)}\right)\right), \quad n \geq 0 .
$$

For each $k<d$, denote elements of $\mathbf{R}^{d-k}$ by $\left(t_{k+1}, t_{k+2}, \ldots, t_{d}\right)$, set $U_{d-1}=$ identity mapping on $L^{2}(\mathbf{R})$, and define for $k<d-1, U_{k}: H\left(\pi_{k}\right) \rightarrow L^{2}\left(\mathbf{R}^{d-k}\right)$ by $U_{k} f\left(t_{k+1}, t_{k+2}, \ldots, t_{d}\right)=U_{k+1}\left(f\left(t_{k+1}\right)\right)\left(t_{k+2}, \ldots, t_{d}\right)$. Set for each $n \geq 0$, $\pi_{n}=U_{0} \pi_{0}^{(n)} U_{0}^{-1}$. Now suppose that $D=t_{k}$, and set $j=k-1$. For each $n \geq 0$, let $Y_{k}^{(n)} \in \mathfrak{m}_{j 1}\left(\lambda_{n}\right) \sim \mathfrak{m}_{j 0}\left(\lambda_{n}\right)$ such that $\lambda\left(Y_{k}^{(n)}\right)=0$ and $B_{\lambda_{n}}\left(X_{k}^{(n)}, Y_{k}^{(n)}\right)=1$. It is easily seen that $Y_{k}^{(n)} \rightarrow Y_{k}^{(0)}$, and that for each $n, U_{j} \pi_{j}^{(n)} U_{j}^{-1}\left(-i Y_{k}^{(n)}\right)=t_{k}$. If $j=0$, we are done. Otherwise, we apply Lemma 2.3 to obtain, for each $n, W^{(n)} \in$ $U\left(g^{k}\left(\lambda_{n}\right)_{c}\right)$ such that $\pi_{j-1}^{(n)}\left(W^{(n)}\right)=\pi_{j}^{(n)}\left(-i Y_{k}^{(n)}\right)$. The construction whereby $W^{(n)}$ is obtained involves only ad $X_{j}^{(n)}, Y_{j}^{(n)}=Y_{k-1}^{(n)} \in \mathfrak{m}_{k 1}\left(\lambda_{n}\right) \cap \operatorname{ker}\left(\lambda_{n}\right) \sim \mathfrak{m}_{k 0}\left(\lambda_{n}\right)$ where $B_{\lambda_{n}}\left(X_{j}^{(n)}, Y_{j}^{(n)}\right)=1, n=0,1,2,3, \ldots$, and we have $Y_{j}^{(n)} \rightarrow Y_{j}^{(0)}$ as well as $\operatorname{ad} X_{j}^{(n)} \rightarrow \operatorname{ad} X_{j}^{(0)}$. Hence it is clear that for some $m, W^{(n)} \in U\left(\mathfrak{g}_{c}\right)^{(m)}, n \geq 0$, and $W^{(n)} \rightarrow W^{(0)}$, and from the definition of $U_{j-1}$ it is clear that for each $n$, $U_{j-1} \pi_{j-1}^{(n)} U_{j-1}^{-1}\left(W^{(n)}\right)=t_{k}$. If $j=1$, then we are done. If $j>1$, then we continue this process applying Lemma 2.3 at each step. This finishes the case $D=t_{k}$. If $D=\partial / \partial t_{k}$, the proof is similar. Q.E.D.

4. A theorem. We now drop the assumption that $\mathfrak{g}$ is nilpotent, that is, let $\mathfrak{g}$ be a real solvable Lie algebra of exponential type, and $G$ a connected, simply connected Lie group with Lie algebra $\mathfrak{g}$. Let $\mathfrak{n}$ be the nilradical of $\mathfrak{g}$, and for the 
remainder of this paper, let $\mathfrak{n}=\mathfrak{n}_{p} \supset \mathfrak{n}_{p-1} \supset \cdots \supset \mathfrak{n}_{0}=(0)$ be a Jordan-Hölder sequence for $\mathfrak{n}$ having the property that for each $t=1,2,3, \ldots, p-1$, if $\left[\mathfrak{g}, \mathfrak{n}_{t}\right] \not \subset \mathfrak{n}_{t}$, then $\left[\mathfrak{g}, \mathfrak{n}_{t+1}\right] \subset \mathfrak{n}_{t+1}$. Let $E$ be the index set and $\left\{\Omega_{\alpha}\right\}_{\alpha \in E}$ the $\operatorname{Ad}^{*}(N)$-invariant partition of $\mathfrak{n}^{*}$ corresponding to this Jordan-Hölder sequence as constructed in the previous section.

Let $\lambda \in \mathfrak{n}^{*}, \mathfrak{p}(\lambda)=\sum_{t} R\left(\lambda, \mathfrak{n}_{t}\right)$. Suppose that there is $A \in R(\lambda, \mathfrak{g}) \sim \mathfrak{n}$, and set $\mathfrak{h}=\mathbf{R} A+\mathfrak{n}, H=\exp (\mathfrak{h})$. It is shown in $[\mathbf{1}]$ that $\mathfrak{p}(\lambda)$ is invariant under ad $A$, and we may extend the equivalence class of $\sigma=\operatorname{ind}(\lambda, \mathfrak{p}(\lambda))$ in $\hat{N}$ to $H$ by setting

$$
(\sigma(\exp s A) f)(y)=f(\exp (-s A) \cdot y \cdot \exp s A) \exp ^{\left\{\frac{1}{2} \operatorname{tr}\left(\operatorname{ad}_{n / \mathfrak{p}\left(\lambda_{n}\right)} A_{n}\right)\right\}}, \quad y \in N
$$

The corresponding extension of $\lambda$ to $\mathfrak{h}$ is obtained by setting $\lambda(A)=0$. Now let $\mathfrak{n}=\mathfrak{n}^{0}(\lambda) \supset \mathfrak{n}^{1}(\lambda) \supset \cdots \supset \mathfrak{n}^{d}(\lambda)=\mathfrak{p}(\lambda)$ be the Kirillov sequence in $\lambda$ as constructed in the previous section, let $X_{k} \in \mathfrak{n}^{k-1}(\lambda) \sim \mathfrak{n}^{k}(\lambda), k=1,2, \ldots, d$, and let $\pi=\pi_{0}$ be the irreducible representation of $N$ corresponding to $\lambda$ as constructed in Theorem 3.5. Then $\pi_{0}=U \sigma U^{-1}$ where $U: H\left(\pi_{0}\right) \rightarrow L^{2}\left(\mathbf{R}^{d}\right)$ is defined by

$$
U f\left(t_{1}, t_{2}, \ldots, t_{d}\right)=f\left(\exp t_{1} X_{1} \cdot \exp t_{2} X_{2} \cdots \exp t_{d} X_{d}\right)
$$

We extend $\pi_{0}$ as indicated above (that is, so as to be isomorphic with the above extension of $\sigma$ ).

Now let $\alpha \in E$ such that $\lambda \in \Omega_{\alpha}$, and suppose that $\left\{\lambda_{n}\right\}_{n=1}^{\infty}$ is a sequence in $\Omega_{\alpha}$ such that $\lambda_{n} \rightarrow \lambda$. By Theorem 3.5, we have a corresponding sequence $\left\{\pi_{n}\right\}_{n=1}^{\infty}$ of irreducible representations of $N$ such that if $D$ is a polynomial differential operator on $\mathbf{R}^{d}$, then there is $W_{n} \in U\left(\mathfrak{n}_{c}\right)^{m}, n=0,1,2, \ldots$, for some $m$, such that $W_{n} \rightarrow W_{0}$ and $\pi_{n}\left(W_{n}\right)=D$ for each $n$. Recall that for each $n$, we have $X_{k}^{(n)} \in \mathfrak{n}^{k-1}\left(\lambda_{n}\right) \sim \mathfrak{n}^{k}\left(\lambda_{n}\right), k=1,2, \ldots, d$ such that $\operatorname{ind}\left(\lambda, \mathfrak{p}\left(\lambda_{n}\right)\right)$ is equivalent to $\pi_{n}$ via the isomorphism

$$
U f\left(t_{1}, t_{2}, \ldots, t_{d}\right)=f\left(\exp t_{1} X_{1}^{(n)} \cdot \exp t_{2} X_{2}^{(n)} \cdots \exp t_{d} X_{d}^{(n)}\right)
$$

and for each $k, X_{k}^{(n)} \rightarrow X_{k}$. Suppose that we have $A_{n} \in R\left(\lambda_{n}, \mathfrak{g}\right) \sim \mathfrak{n}, n=$ $1,2,3, \ldots$, such that $A_{n} \rightarrow A$, and set $h_{n}=\mathbf{R} A_{n}+\mathfrak{n}, H_{n}=\exp h_{n}$ for each $n$. Extend $\pi_{n}$ to $H_{n}$, as above, so that the corresponding extension of $\lambda_{n}$ is obtained by setting $\lambda_{n}\left(A_{n}\right)=0$. Let the algebra $\mathfrak{D}$ of polynomial differential operators on $\mathbf{R}^{d}$ have the (obvious) filtration $\mathfrak{D}^{(0)} \subset \mathfrak{D}^{(1)} \subset \mathfrak{D}^{(2)} \subset \cdots$ so that $D \in \mathfrak{D}^{(m)}$ if and only if there is a polynomial $P$ of degree $\leq m$ such that $D=P\left(t_{1}, t_{2}, \ldots, t_{d}, \partial / \partial t_{1}, \partial / \partial t_{2}, \ldots, \partial / \partial t_{d}\right)$, and for each $m$, let $\mathfrak{D}^{(m)}$ have the usual topology as a finite dimensional vector space over $\mathbf{C}$.

LEMMA 4.1. There is an integer $m>0$ such that $\pi_{0}(A) \in \mathfrak{D}^{(m)},\left\{\pi_{n}\left(A_{n}\right)\right\}_{n=0}^{\infty}$ $\subset \mathfrak{D}^{(m)}$, and $\pi_{n}\left(A_{n}\right) \rightarrow \pi_{0}(A)$ in $\mathfrak{D}^{(m)}$.

PrOOF. Clearly we may assume that $d>0$. Let us use the notation $T=$ $\left(t_{1}, t_{2}, \ldots, t_{d}\right), U=\left(u_{1}, u_{2}, \ldots, u_{p}\right)$ and $Z=\left(z_{11}, z_{12}, \ldots, z_{i j}, \ldots, z_{p p}\right)$ for elements of $\mathbf{R}^{d}, \mathbf{R}^{p}$, and $\mathbf{R}^{p^{2}}$, respectively, and denote the objects associated with $\pi_{0}$ by $\lambda_{0}, A_{0}$, etc. For each $n \geq 0$, let $\left\{X_{k}^{(n)}\right\}_{k=d+1}^{p}$ be a basis of $\mathfrak{p}\left(\lambda_{n}\right)$ such that $X_{k}^{(n)} \rightarrow X_{k}^{(0)}, d<k \leq p$, and for each $i, j=1,2, \ldots, p$ and $s \in \mathbf{R}$, let $a_{i j}^{(n)}(s)$ denote 
the coefficient of $X_{j}^{(n)}$ in the expansion of $e^{s \text { ad } A_{n}}\left(X_{i}^{(n)}\right)$ in terms of the ordered basis $X_{1}^{(n)}, X_{2}^{(n)}, \ldots, X_{p}^{(n)}$ of $\mathfrak{g}$. Denote the element $\left(a_{11}^{(n)}(s), a_{12}^{(n)}(s), \ldots, a_{i j}^{(n)}(s), \ldots\right.$, $\left.a_{p p}^{(n)}(s)\right)$ of $\mathbf{R}^{p^{2}}$ by $a^{(n)}(s)$.

By the Campbell-Hausdorff formula, we have for each $n$, polynomials $P_{1}^{(n)}, P_{2}^{(n)}$, $\ldots, P_{p}^{(n)}$ in $T$ such that

$$
\prod_{j=1} \exp t_{j} X_{j}^{(n)}=\exp \left(\sum_{j=1}^{p} P_{j}^{(n)}(T) X_{j}^{(n)}\right) .
$$

Let $q>0$ and such that $N$ is step $q$. Then for each $n, j, \operatorname{deg}\left(P_{j}^{(n)}\right)<q$, and the coefficients of $P_{j}^{(n)}$ depend only on the structure constants $\left(b_{k}^{i j}\right)^{(n)},\left[X_{i}^{(n)}, X_{j}^{(n)}\right]=$ $\sum\left(b_{k}^{i j}\right)^{(n)} X_{k}^{(n)}$. Clearly for each $i, j, k,\left(b_{k}^{i j}\right)^{(n)} \rightarrow\left(b_{k}^{i j}\right)^{(0)}$ and hence $P_{j}^{(n)} \rightarrow P_{j}^{(0)}$ in the vector space $\mathbf{C}[T]^{(q)}, 1 \leq j \leq p$. Now let the polynomials $\tilde{P}_{j}^{(n)}$ in $T$ and $Z$ be defined by $\tilde{P}_{j}^{(n)}(T, Z)=\sum_{i} P_{i}^{(n)}(T) z_{i j}$; then we have

$$
\exp -s A_{n}\left[\prod_{j=1}^{d} \exp t_{j} X_{j}^{(n)}\right] \exp s A_{n}=\exp \sum_{j=1}^{p} \tilde{P}_{j}^{(n)}\left(T, a^{(n)}(s)\right) X_{j}^{(n)},
$$

$$
s \in \mathbf{R}, n=0,1,2,3, \ldots .
$$

On the other hand, there are polynomials $R_{j}^{(n)}, 1 \leq j \leq p$, in $U$ such that

$$
\exp \sum_{j=1}^{p} u_{j} X_{j}^{(n)}=\exp \sum_{j>d} R_{j}^{(n)}(U) X_{j}^{(n)} \cdot \prod_{j=1}^{d} \exp R_{j}^{(n)}(U) X_{j}^{(n)} .
$$

As with $P_{j}^{(n)}$, we see that for each $n, j, \operatorname{deg}\left(R_{j}^{n}\right) \leq q$, and for each $j, R_{j}^{(n)} \rightarrow R_{j}^{(0)}$ in $\mathbf{C}[U]^{(q)}$. Now let $Q_{j}^{(n)}=R_{j}^{(n)}\left(\tilde{P}_{1}^{(n)}, \ldots, \tilde{P}_{p}^{(n)}\right), 1 \leq j \leq p$. Then $Q_{j}^{(n)} \rightarrow Q_{j}^{(0)}$ in $\mathbf{C}[T, Z]^{\left(q^{2}\right)}$ and from the definition of $\pi_{n}$ we have, for each $\phi \in C^{\infty}\left(\pi_{n}\right)$,

$$
\begin{aligned}
\left(\pi_{n}\left(A_{n}\right) \phi\right)(T)= & \left.\frac{d}{d s}\right|_{s=0} \exp ^{i \sum_{i>d} Q_{j}^{(n)}\left(T, a^{(n)}(s)\right) \lambda_{n}\left(X_{j}^{(n)}\right)} \\
& \cdot \phi\left(Q_{1}^{(n)}\left(T, a^{(n)}(s)\right), Q_{2}^{(n)}\left(T, a^{(n)}(s)\right), \ldots, Q_{d}^{(n)}\left(T, a^{(n)}(s)\right)\right) \\
& \cdot \exp ^{\left\{-\frac{1}{2} \operatorname{tr}\left(\operatorname{ad}_{n / p\left(\lambda_{n}\right)} A_{n}\right)\right\}}
\end{aligned}
$$

for each $n$. Let $\tilde{Q}_{j}^{(n)}$ be the polynomial in $\mathbf{C}[T]^{(q)}$ such that

$$
\left.\frac{d}{d s}\right|_{s=0} Q_{j}^{(n)}\left(T, a^{(n)}(s)\right)=\tilde{Q}_{j}^{(n)}(T), \quad 1 \leq j \leq p, n \geq 0 .
$$

Note that $a_{i j}^{(n)}(0)=\delta_{i j}$ for each $i, j$ and $n$, and for each $i, j, d /\left.d s\right|_{s=0} a_{i j}^{(n)}(s) \rightarrow$ $d /\left.d s\right|_{s=0} a_{i j}^{(0)}(s)$, whence $\tilde{Q}_{j}^{(n)} \rightarrow \tilde{Q}_{j}^{(0)}$. Since for each $n$,

$$
\pi_{n}\left(A_{n}\right)=i \sum_{j>d} \tilde{Q}_{j}^{(n)}(T) \lambda_{n}\left(X_{j}^{(n)}\right)+\sum_{j=1}^{d} \tilde{Q}_{j}^{(n)}(T) \frac{\partial}{\partial t_{j}}-\frac{1}{2} \operatorname{tr}\left(\operatorname{ad} n / P\left(\lambda_{n}\right) A_{n}\right)
$$

the result follows. Q.E.D.

For each $n>0$, define $A_{n}^{*}$ in $\mathfrak{h}_{n}^{*}$ by setting $A_{n}^{*}\left(A_{n}\right)=1,\left.A_{n}^{*}\right|_{\mathfrak{n}} \equiv 0$. 
COROLLARY 4.2. There is an integer $m>0$ and for each $n=0,1,2, \ldots$, there is $W_{n} \in U\left(\left(\mathfrak{h}_{n}\right)_{c}\right)$ such that $\left\{W_{n}\right\}_{n=0}^{\infty} \subset U\left(\mathfrak{g}_{c}\right)^{(m)}, W_{n} \rightarrow W_{0}$ in $U\left(\mathfrak{g}_{c}\right)^{(m)}$, and such that for any real sequence $\left\{c_{n}\right\}_{n=0}^{\infty},\left(\chi_{c_{n}} \otimes \pi_{n}\right)\left(W_{n}\right)=c_{n}$ where $\chi_{c_{n}}$ is the character of $H_{n}$ with differential $i c_{n} A_{n}^{*}$.

PROOF. By Lemma 4.1, for each $n$ we may write $\pi_{n}\left(A_{n}\right)=\sum_{\mu} a_{\mu}^{(n)} D_{\mu}$ where $\left\{D_{\mu}\right\}$ is a finite collection of polynomial differential operators and for each $\mu$, $\left\{a_{\mu}^{(n)}\right\}_{n=0}^{\infty}$ is a sequence of complex numbers such that $a_{\mu}^{(0)}=\lim _{n} a_{\mu}^{(n)}$. By Theorem 3.5, for each $\mu$, there is a sequence $\left\{V_{\mu}^{(n)}\right\}_{n=0}^{\infty} \subset U\left(\mathfrak{n}_{c}\right)^{\left(m_{\mu}\right)}$ such that $V_{\mu}^{(n)} \rightarrow V_{\mu}^{(0)}$ in $U\left(\mathfrak{n}_{c}\right)^{\left(m_{\mu}\right)}$ and such that for each $n, \pi_{n}\left(V_{\mu}^{(n)}\right)=D_{\mu}$. Thus $\pi_{n}\left(A_{n}-\sum_{\mu} a_{\mu}^{(n)} V_{\mu}^{(n)}\right)=0, n=0,1,2, \ldots$, and we may take $m=\max _{\mu}\left\{m_{\mu}\right\}$ and

$$
W_{n}=-i\left(A_{n}-\sum_{\mu} a_{\mu}^{(n)} V_{\mu}^{(n)}\right), \quad n=0,1,2, \ldots \quad \text { Q.E.D. }
$$

Let $K(G)$ be the space of all closed, connected subgroups of $G$ (with the compactopen topology), and let $S(G)$ be the space of all pairs $(\rho, H)$ where $H \in K(G)$ and $\rho$ is an unitary equivalence class of representations of $H$ with the topology of Fell (cf. [5]). Let $K_{N}(G)$ be the set of all $H \in K(G)$ such that $N \subset H$, and $S_{N}(G)$ the set of all $(\rho, H) \in S(G)$ such that $H \in K_{N}(G)$ and $\rho \in \hat{H}$. For each $H \in K_{N}(G)$ we have a topological embedding of $\hat{H}$ in $S_{N}(G)$. The proof that $\eta_{G}$ is continuous (cf. [10, Proposition 2]) is easily generalized to show that the mapping $\Theta: \mathfrak{g}^{*} \times K_{N}(G) \rightarrow S_{N}(G)$ given by

$$
\Theta((l, H))=\left(\eta_{H}\left(\operatorname{Ad}^{*}(H)\right)\left(l l_{\mathfrak{h}}\right), H\right)
$$

is continuous, where $\mathfrak{g}^{*} \times K_{N}(G)$ has the product topology. If $(\rho, H) \in S_{N}(G)$ we denote the $\operatorname{Ad}^{*}(H)$-orbit $\eta_{H}^{-1}(\rho)$ by $O_{\rho}$, and if $J \subset H, \mathfrak{j}=\log (J)$, let $\left.O_{\rho}\right|_{j}=$ $\left\{\left.l\right|_{j} \mid l \in O_{\rho}\right\}$.

The following two facts are well-known consequences of the general theory (cf. [4 and 5$]$ ).

LEMma 4.3. Let $(\rho, H) \in S_{N}(G)$ and let $J \in K_{N}(G)$ be a subgroup of $H$. Then the set of all $\sigma \in \hat{J}$ such that $\left.O_{\sigma} \subset O_{\rho}\right|_{\log (J)}$ is a dense subset of $\operatorname{Sp}\left(\left.\rho\right|_{J}\right)$.

LEMMA 4.4. Let $(\rho, H) \in S_{N}(G)$, and let $\left\{\left(\rho_{n}, H_{n}\right)\right\}_{n=1}^{\infty}$ be a sequence in $S_{N}(G)$ such that $\left(\rho_{n}, H_{n}\right) \rightarrow(\rho, H)$. Let $J \in K_{N}(G)$ and for each $n, J_{n} \in K_{N}(G)$ such that $J \subset H, J_{n} \subset H_{n}$, and $J_{n} \rightarrow J$. Let $(\sigma, J) \in S_{N}(G)$ such that $O_{\sigma} \subset$ $\left.O_{\rho}\right|_{\log (J)}$. Then for each $n$, there is $\sigma_{n} \in \hat{J}_{n}$ such that $\left.O_{\sigma_{n}} \subset O_{\rho_{n}}\right|_{\log \left(J_{n}\right)}$ and such that the sequence $\left\{\left(\sigma_{n}, J_{n}\right)\right\}_{n=1}^{\infty}$ converges to $(\sigma, J)$.

We define a partition of $S_{N}(G)$ as follows. For each $(\rho, H) \in S_{N}(G)$, let $\alpha(\rho)$ be the smallest index in $E$ such that $O_{\rho} \ln _{\mathfrak{n}} \cap \Omega_{\alpha} \neq\{\phi\}$. For each $\alpha \in E$, let

$$
\tilde{V}_{\alpha}=\left\{(\rho, H) \in S_{N}(G) \mid \alpha(\rho)=\alpha\right\} .
$$

From Brown's Theorem [3] and Lemma 4.3 above it follows that $(\rho, H) \in \tilde{V}_{\alpha}$ if and only if $\operatorname{Sp}\left(\left.\rho\right|_{N}\right) \cap \eta_{N}\left(\Omega_{\alpha}\right) \neq\{\phi\}$ and $\operatorname{Sp}\left(\left.\rho\right|_{N}\right) \cap \eta_{N}\left(\Omega_{\beta}\right)=\{\phi\}$ for all $\beta<\alpha$. For each $\alpha$, set $V_{\alpha}=\tilde{V}_{\alpha} \cap \hat{G}$, and $U_{\alpha}=\eta_{G}^{-1}\left(V_{\alpha}\right)$. Then $U_{\alpha}=\left\{O \in \mathfrak{g}^{*} / \operatorname{Ad}^{*}(G)|O|_{n} \cap \Omega_{\alpha} \neq\right.$ $\{\phi\}$ and $\left.O\right|_{\mathfrak{n}} \cap \Omega_{\beta}=\{\phi\}$, for all $\left.\beta<\alpha\right\}$. 
LEMMA 4.5. For each $\alpha, \tilde{V}_{\alpha}$ is open in $\bigcup_{\beta \geq \alpha} \tilde{V}_{\beta}$. If $\alpha_{0}$ is the smallest element of $E$, then $\tilde{V}_{\alpha_{0}}$ is dense in $S_{N}(G)$.

PROOF. Let $(\rho, H) \in \tilde{V}_{\alpha}$ and suppose that $\left\{\left(\rho_{n}, H_{n}\right)\right\}_{n=1}^{\infty}$ is a sequence in $\bigcap_{\beta \geq \alpha} \tilde{V}_{\rho}$ such that $\left(\rho_{n}, H_{n}\right) \rightarrow(\rho, H)$. Let $\sigma_{0} \in \operatorname{Sp}\left(\left.\rho\right|_{N}\right)$ such that $\left.O_{\sigma_{n}} \subset O_{\rho}\right|_{n} \cap$ $\Omega_{\alpha}$. By Lemma 4.4, there is $\left\{\sigma_{n}\right\} \subset \hat{N}$ such that $\sigma_{n} \rightarrow \sigma$ and for each $n, O_{\sigma_{n}} \subset$ $O_{\rho_{n}} \mid N$. By Brown's Theorem, $O_{\sigma_{n}} \rightarrow O_{\sigma}$. Since $\left\{O_{\sigma_{n}}\right\} \subset \cup_{\beta \geq \alpha} \Omega_{\alpha}$ and $\Omega_{\alpha}$ is open in $\bigcup_{\beta \geq \alpha} \Omega_{\beta},\left\{O_{\sigma_{n}}\right\}$ is eventually in $\Omega_{\alpha}$, thus $\left\{\left(\rho_{n}, H_{n}\right)\right\}_{n=1}^{\infty}$ is eventually in $\tilde{V}_{\alpha}$.

Let $\alpha_{0}$ be the minimal element of $E$ and let $(\rho, H) \in S_{N}(G), \mathfrak{h}=\log (H)$. The set $\left\{O \in \mathfrak{h}^{*} / \operatorname{Ad}^{*}(H)|O|_{\mathfrak{n}} \cap \Omega_{\alpha_{0}} \neq\{\phi\}\right\}$ is dense in $\mathfrak{h}^{*} / \operatorname{Ad}^{*}(H)$, hence the set $\left\{\rho \in \hat{H}\left|O_{\rho}\right|_{\mathfrak{n}} \cap \Omega_{\alpha} \neq\{\phi\}\right\}$ is dense in $\hat{H}$ (by continuity of $\eta_{H}$ ). The embedding of this set in $S_{N}(G)$ is contained in $\tilde{V}_{\alpha_{0}}$ and $(\rho, H)$ is contained in its closure.

Let $(\rho, H) \in S_{N}(G), \mathfrak{h}=\log (H)$, and let $\left.\lambda \in O_{\rho}\right|_{\mathfrak{n}}$. Let $\mathfrak{j}=R(\lambda, h)+\mathfrak{n}$, $J=\exp (\mathfrak{j})$, and let $\sigma \in \hat{J}$ such that $\left.O_{\sigma} \subset O_{\rho}\right|_{\mathfrak{j}}$. Suppose that $\mathfrak{j} \neq \mathfrak{n}$, and let $\left\{A_{1}, A_{2}, \ldots, A_{r}\right\} \subset R(\lambda, h)$ be a basis for $\mathfrak{j} \bmod \mathfrak{n}$. Define $A_{j}^{*} \in \mathfrak{h}^{*}$ by $A_{j}^{*}\left(A_{i}\right)=\delta_{i j}$ and $\left.A_{j}^{*}\right|_{\mathfrak{n}} \equiv 0$. Recall then that $\left.\sigma\right|_{N} \in \hat{N}$, and that if $\lambda$ is extended to $j^{*}$ by setting $\lambda\left(A_{j}\right)=0,1 \leq j \leq r$, then there is a unique $t=\left(t_{1}, t_{2}, \ldots, t_{r}\right) \in \mathbf{R}^{r}$ such that $\lambda+\sum_{j=1}^{r} t_{j} A_{j}^{*} \in O_{\sigma}$. For any $j, 1 \leq j \leq r$, if $\nu=\left.\sigma\right|_{N}$ is extended to $H_{j}=\exp \left(\mathbf{R} A_{i}+N\right)$ by formula $(2)$, and $\chi_{t_{i}}$ is the character of $H_{j}$ having differential $i t_{j}\left(\left.A_{j}^{*}\right|_{h_{j}}\right)$, then $\left.\sigma\right|_{H_{j}}=\chi_{t_{i}} \otimes \nu$.

LEMMA 4.6. Let $\alpha \in E$ and let $\lambda_{n} \in \Omega_{\alpha}, n=0,1,2, \ldots$, such that the sequence $\left\{\lambda_{n}\right\}_{n=1}^{\infty}$ converges to $\lambda_{0}$, for each $n \geq 0$, let $A_{n} \in R\left(\lambda_{n}, \mathfrak{g}\right) \sim \mathfrak{n}, \mathfrak{h}_{n}=\mathbf{R} A_{n}+\mathfrak{n}$, $H_{n}=\exp \left(\mathfrak{h}_{n}\right)$, extend $\lambda_{n}$ to $\mathfrak{h}_{n}$ by setting $\lambda_{n}\left(A_{n}\right)=0$, define $A_{n}^{*} \in \mathfrak{h}_{n}^{*}$ by $A_{n}^{*}\left(A_{n}\right)=$ $1,\left.A_{n}^{*}\right|_{n} \equiv 0$, let $t_{n} \in \mathbf{R}$, and let $\rho_{n}=\eta_{H_{n}}^{-1} \operatorname{Ad}^{*}\left(H_{n}\right)\left(\lambda_{n}+t_{n} A_{n}^{*}\right)$. Assume that $A_{n} \rightarrow A_{0}$ as $n \rightarrow \infty$. Then $\left(\rho_{n}, H_{n}\right) \rightarrow\left(\rho_{0}, H_{0}\right)$ if and only if $t_{n} \rightarrow t_{0}$.

PROOF. We need only prove the "only if" part. Suppose that $\left(\rho_{n}, H_{n}\right) \rightarrow$ $\left(\rho_{0}, H_{0}\right)$. Let $\pi_{0}$ an irreducible representation corresponding to $\lambda_{0}$ and let $\left\{\pi_{n}\right\}_{n=1}^{\infty}$ a sequence of representations corresponding to $\left\{\lambda_{n}\right\}_{n=1}^{\infty}$ as obtained in Theorem 3.5, so that $\left.\pi_{n} \in \rho_{n}\right|_{N}, n \geq 0$. Extend $\pi_{n}$ to $H_{n}$ as in formula (2) so as to correspond to $\lambda_{n}$, and let $\chi_{n}$ be the character of $H_{n}$ such that $\gamma_{n}=\chi_{n} \otimes \pi_{n} \in \rho_{n}$. Then by Corollary 4.2, there is $m>0$ and $\left\{W_{n}\right\}_{n=0}^{\infty} \subset U\left(\mathfrak{g}_{c}\right)^{(m)}$ such that $W_{n} \rightarrow W_{0}$ and such that for each $n, W_{n} \in U\left(\left(\mathfrak{h}_{n}\right)_{c}\right)$ and $\gamma_{n}\left(W_{n}\right)=t_{n}$. Now the general theory implies that $t_{n} \rightarrow t_{0}$. To see this, let $\Psi_{0} \in C_{c}^{\infty}(G)$ and $v_{0} \in H\left(\gamma_{0}\right)$ such that $\left\langle\gamma_{0}\left(\Psi_{0}\right) v_{0}, v_{0}\right\rangle \neq 0$. For each $n$, let $\Gamma_{n}$ be the representation of $C_{s}^{*}(G)$ lifted from $\gamma_{n}$. Note that any $\Psi \in C_{c}^{\infty}(G)$ defines in a natural way an element $\tilde{\Psi}$ in $C_{s}^{*}(G)$ by setting $\tilde{\Psi}((K, x))=\Psi(x), K \in K(G), x \in K$ such that for any $v \in H\left(\gamma_{n}\right)$, $\left\langle\Gamma_{n}(\tilde{\Psi}) v, v\right\rangle=\left\langle\gamma_{n}(\Psi) v, v\right\rangle$. Now let $V_{1}, V_{2}, \ldots, V_{q} \in U\left(\mathfrak{g}_{c}\right)$ such that for each $n$, $W_{n}=\sum_{j=1}^{q} a_{j}^{(n)} V_{j}$ with $a_{j}^{(n)} \in C, 1 \leq j \leq q$, for each $j, a_{j}^{(0)}=\lim _{n} a_{j}^{(n)}$. Set $\Psi_{j}=V_{j} \Psi_{0}, 1 \leq j \leq q$. Then by Lemma 2.2 of [4], there is, for each $n>0$, $v_{n} \in H\left(\gamma_{n}\right)$ such that $\left\langle\Gamma_{n}\left(\tilde{\Psi}_{j}\right) v_{n}, v_{n}\right\rangle \rightarrow\left\langle\Gamma_{0}\left(\tilde{\Psi}_{j}\right) v_{0}, v_{0}\right\rangle$ as $n \rightarrow \infty, 0 \leq j \leq q$. Thus $\left\langle\Gamma_{n}\left(W_{n} \Psi_{0}\right) v_{n}, v_{n}\right\rangle \rightarrow\left\langle\Gamma_{0}\left(W_{0} \Psi_{0}\right) v_{0}, v_{0}\right\rangle$ as $n \rightarrow \infty$, and we have

$$
t_{n}=-\frac{\left\langle\gamma_{n}\left(W_{n} \Psi_{0}\right) v_{n}, v_{n}\right\rangle}{\left\langle\gamma_{n}\left(\Psi_{0}\right) v_{n}, v_{n}\right\rangle} \rightarrow-\frac{\left\langle\gamma_{0}\left(W_{0} \Psi_{0}\right) v_{0}, v_{0}\right\rangle}{\left\langle\gamma_{0}\left(\Psi_{0}\right) v_{0}, v_{0}\right\rangle}=t_{0} . \quad \text { Q.E.D. }
$$

For each $\alpha \in E$, set $\tilde{U}_{\alpha}=\Theta^{-1}\left(\tilde{V}_{\alpha}\right)$. 
THEOREM 4.7. $\left.\Theta\right|_{\tilde{U}_{d}}: \tilde{U}_{\alpha} \rightarrow \tilde{V}_{\alpha}$ is open, for each $\alpha \in E$.

ProOF. Let $\left(\rho_{0}, H_{0}\right) \in \tilde{V}_{\alpha}$, and suppose that $\left\{\left(\rho_{n}, H_{n}\right)\right\}_{n=1}^{\infty}$ is a sequence in $\tilde{V}_{\alpha}$ which converges to $\left(\rho_{0}, H_{0}\right)$. Let $\mathfrak{h}_{n}=\log \left(H_{n}\right), n=0,1,2, \ldots$, and let $l_{0} \in \mathfrak{g}^{*}$ such that $l l_{\mathfrak{h}} \in O_{\rho_{0}}$. It is enough to show that there is a subsequence $\left\{\left(\rho_{k}, H_{k}\right)\right\}_{k=1}^{\infty}$ of $\left\{\left(\rho_{n}, H_{n}\right)\right\}_{n=1}^{\infty}$ and a corresponding sequence $\left\{l_{k}\right\}_{k=1}^{\infty}$ in $\mathfrak{g}^{*}$ such that $\left.l_{k}\right|_{\mathfrak{h}_{k}} \in O_{\rho_{k}}$ for each $k$ and $l_{k} \rightarrow l_{0}$. Note that we may assume that $\lambda_{0}=\left.l_{0}\right|_{n} \in \Omega_{\alpha}$. Let $\nu \in \hat{N}$ such that $\lambda_{0} \in O_{\nu}$. By Lemma 4.3, there is $\nu_{n} \in \hat{N}$ such that $\left.O_{\nu_{n}} \subset O_{\rho_{n}}\right|_{n}$, $n=1,2,3, \ldots$, and such that the sequence $\left\{\nu_{n}\right\}_{n=1}^{\infty}$ converges to $\nu$. Thus we have $\left.\lambda_{n} \in O_{\nu_{n}} \subset O_{\rho_{n}}\right|_{n}, n=1,2,3, \ldots$, such that $\left\{\lambda_{n}\right\}$ converges to $\lambda_{0}$. Now by restriction to a subsequence, we may assume that $\operatorname{dim}\left(\mathfrak{h}_{n}\right)=m, n=0,1,2, \ldots$, and since $\Omega_{\alpha}$ is open in $\bigcup_{\beta \geq \alpha} \Omega_{\beta}$ and $\left\{\lambda_{n}\right\} \in \bigcup_{\beta \geq \alpha} \Omega_{\alpha}$, we may assume that $\lambda_{n} \in \Omega_{\alpha}$ for all $n$. We proceed by induction on $\operatorname{dim}\left(\mathfrak{h}_{n} / \mathfrak{n}\right)=m-p$.

The case $m-p=0$ is now trivial due to the above, so assume that $m>p$ and that the theorem is valid for sequences in $S_{N}(G)$ whose subgroup have dimension less than $m$. Let $\left\{\lambda_{k}\right\}_{k=1}^{\infty}$ be a subsequence of $\left\{\lambda_{n}\right\}_{n=1}^{\infty}$ such that for some subalgebra $\mathfrak{j}_{0}$, the sequence $\mathfrak{j}_{k}=R\left(\lambda_{k}, \mathfrak{h}_{k}\right)+\mathfrak{n}, k=1,2,3, \ldots$, converges to $\mathfrak{j}_{0}$. Let $J_{k}=$ $\exp \left(\mathfrak{j}_{k}\right), k \geq 0$. By Lemma 4.3, we have $\sigma_{k} \in \hat{J}_{k}, k=0,1,2, \ldots$, such that $l_{0}\left|j_{0} \in O_{\sigma_{0}}, O_{\sigma_{k}} \subset O_{\rho_{k}}\right|_{j_{k}}, k \geq 1$, and the sequence $\left\{\left(\sigma_{k}, J_{k}\right)\right\}_{k=1}^{\infty}$ converges to $\left(\sigma_{0}, J_{0}\right)$. Suppose that $\operatorname{dim} J_{0}<m$. By induction there is $l_{k} \in \mathfrak{g}^{*}$ such that $\left.l_{k}\right|_{j_{k}} \in O_{\sigma_{k}}, k=1,2,3, \ldots$, and such that the sequence $\left\{l_{k}\right\}_{k=1}^{\infty}$ converges to $l_{0}$. Now if $\rho_{k}: \mathfrak{h}_{k}^{*} \rightarrow \mathfrak{j}_{k}^{*}$ is the restriction mapping, then $\rho_{k}^{-1}\left(O_{\sigma_{k}}\right) \subset O_{\rho_{k}}, k \geq 1$ (cf. [1, Chapter II, §4.2]). Therefore $\left.l_{k}\right|_{\mathfrak{h}_{k}} \in O_{\rho_{k}}$, and we are done. Hence by induction we have reduced to the case $\mathfrak{j}_{k}=\mathfrak{h}_{k}, k \geq 0$.

For each $k$, let $\left\{A_{1}^{(k)}, A_{2}^{(k)}, \ldots, A_{r}^{(k)}\right\} \subset R\left(\lambda_{k}, \mathfrak{h}_{k}\right)$ be a basis for $\mathfrak{h}_{k} \bmod \mathfrak{n}$ such that for each $1 \leq j \leq r, A_{j}^{(0)}=\lim _{k} A_{j}^{(k)}$. Extending $\lambda_{k}$ to $\mathfrak{h}_{k}$ by setting $\lambda_{k}\left(A_{j}^{(k)}\right)=$ $0,1 \leq j \leq r$, let $t_{1}^{(k)}, t_{2}^{(k)}, \ldots, t_{r}^{(k)}$ be real numbers such that $\lambda_{k}+\sum_{j=1}^{r} t_{j}^{(k)} A_{k}^{(k) *} \in$ $O_{\rho_{k}}$ (where $A_{j}^{(k)^{*}}$ is defined by $A_{j}^{(k)^{*}}\left(A_{i}^{(k)}\right)=\delta_{i j},\left.A_{j}^{(k)^{*}}\right|_{\mathrm{n}} \equiv 0$ ). For each $j, i \leq$ $j \leq r$, apply Lemma 4.6 to the sequence $\left\{\left(\left.\rho_{k}\right|_{\exp \left(\mathbf{R} A_{j}^{(k)}+N\right)}, \exp \left(\mathbf{R} A_{j}^{(k)}+N\right)\right)\right\}_{k=1}^{\infty}$ which converges to $\left(\left.\rho_{0}\right|_{\exp \left(\mathbf{R} A_{j}^{0}+N\right)}, \exp \left(\mathbf{R} A_{j}^{0}+N\right)\right)$, and we obtain $t_{j}^{(0)}=\lim _{k} t_{j}^{(k)}$. Since $A_{j}^{(k)} \rightarrow A_{j}^{(0)}, 1 \leq j \leq q$, it is clear that we may extend $\lambda_{k}+\sum_{j=1}^{q} t_{j}^{(k)} A_{j}^{(k)}$ * $^{*}$ to an element $l_{k} \in \mathfrak{g}^{*}$ such that the sequence $\left\{l_{k}\right\}_{k=1}^{\infty}$ converges to $l_{0}$. This finishes the proof. Q.E.D.

The following corollary is immediate.

COROLLARY 4.8. $\left.\eta_{G}\right|_{U_{a}}: U_{\alpha} \rightarrow V_{\alpha}$ is a homeomorphism, for each $\alpha$.

Note that for each $\alpha \in E$, the dimensions of the orbits in $U_{\alpha}$ may vary, and the relative topology in $U_{\alpha}$ may not be $T_{1}$. Indeed, if $N$ is abelian, then $E=\left\{\alpha_{0}\right\}$ and $U_{\alpha_{0}}=\mathfrak{g}^{*}$. Examples indicate that for each $\alpha \in E$, there is a finite partition of $U_{\alpha}$, each element of which is $T_{2}$. Finally, the subsets $U_{\alpha}$ may be describable as Zariski-open subsets of algebraic varieties in $\mathfrak{g}^{*}$.

The author would like to thank his thesis advisor, Professor Richard Penney, for his help and advice. The author's indebtedness to him goes far beyond the present work. Thanks go also to Professor Jeffrey Fox for some instructive and 
stimulating conversations and to the referee for his attention to this work and his helpful suggestions.

\section{REFERENCES}

1. P. Bernat et al., Représentations des groups de Lie résoluble, Dunod, Paris, 1972.

2. J. Boidol, *-regularity of exponential Lie groups, Invent. Math. 56 (1980), 231-238.

3. I. Brown, Dual topology of a nilpotent Lie group, Ann. Sci. Ecole Norm. Sup. 6 (1973), 407-411.

4. J. M. G. Fell, Weak containment and induced representations of groups, Canad. J. Math. 14 (1964), 237-268.

5. _ Weak containment and induced representations of groups. II, Trans. Amer. Math. Soc. 110 (1964), 424-447.

6. H. Fujiwara, Sur le dual d'un groupe de Lie résoluble exponential, J. Math. Soc. Japan 36 (1984), 629-636.

7. K. T. Joy, A description of the topology on the dual space of a nilpotent Lie group, Pacific J. Math. 112 (1984), 135-139.

8. A. A. Kirillov, Unitary representations of nilpotent Lie groups, Russian Math. Surveys 17 (1962), 53-104.

9. N. V. Pedersen, On the characters of exponential solvable Lie groups, Ann. Sci. Ecole Norm. Sup. (4) 17 (1984), 1-29.

10. L. Pukanszky, On the unitary representations of exponential groups, J. Funct. Anal. 2 (1968), 73-113.

Department of Mathematics and Computer SCience, Saint Louis University, ST. LOUIS, MISSOURI 63103 